\title{
Effects of Reduced Carbohydrate Intake after Sprint Exercise on Breath Acetone Level
}

\author{
Naoki Ota, Hiroto Ito and Kazushige Goto *[D
}

Graduate School of Sport and Health Science, Ritsumeikan University, Kusatsu, Shiga 525-8577, Japan; sh0120hp@gmail.com (N.O.); actarmy.jr.riemann2357@gmail.com (H.I.)

* Correspondence: kagoto@fc.ritsumei.ac.jp; Tel./Fax: +81-77-599-4127

\begin{abstract}
Assessment of breath acetone level may be an alternative procedure to evaluate change in fat metabolism. The purpose of the present study was to investigate the effect of insufficient carbohydrate $(\mathrm{CHO})$ intake after sprint exercise on breath acetone level during post-exercise. Nine subjects conducted two trials, consisting of either reduced $\mathrm{CHO}$ trial (LOW trial) or normal $\mathrm{CHO}$ trial (NOR trial). In each trial, subjects visited to laboratory at 7:30 following an overnight fast to assess baseline breath acetone level. They commenced repeated sprint exercise from 17:00. After exercise, isoenergetic meals with different doses of $\mathrm{CHO}$ (LOW trial; $18 \%$ for $\mathrm{CHO}, 27 \%$ for protein, $55 \%$ for fat, NOR trial; $58 \%$ for $\mathrm{CHO}, 14 \%$ for protein, $28 \%$ for fat) were served. Breath acetone level was also monitored immediately before and after exercise, $1 \mathrm{~h}, 3 \mathrm{~h}, 4 \mathrm{~h}$, and $15 \mathrm{~h}$ (on the following morning) after completing exercise. A significant higher breath acetone level was observed in LOW trial than in NOR trial $4 \mathrm{~h}$ after completion of exercise (NOR trial; $0.66 \mathrm{ppm}$, LOW trial; $0.9 \mathrm{ppm}$ ). However, breath acetone level did not differ on the following morning between two trials. Therefore, $\mathrm{CHO}$ intake following an exhaustive exercise affects breath acetone level during early phase of post-exercise.
\end{abstract}

Keywords: breath acetone; carbohydrate; fat metabolism; substrate utilization; ketone body

check for updates

Citation: Ota, N.; Ito, H.; Goto, K. Effects of Reduced Carbohydrate Intake after Sprint Exercise on Breath Acetone Level. Nutrients 2021, 13, 58. https://dx.doi.org/10.3390/nu13010058

Received: 4 November 2020

Accepted: 24 December 2020

Published: 27 December 2020

Publisher's Note: MDPI stays neutral with regard to jurisdictional claims in published maps and institutional affiliations.

Copyright: () 2020 by the authors. Licensee MDPI, Basel, Switzerland. This article is an open access article distributed under the terms and conditions of the Creative Commons Attribution (CC BY) license (https: / / creativecommons.org/ licenses/by/4.0/).

\section{Introduction}

Muscle glycogen content has been recognized to be one of critical factors for determining endurance exercise performance [1-4]. In addition, a single bout of $30 \mathrm{~s}$ maximal cycle sprint exercise caused 35\% of reduction of muscle glycogen content [5]. Therefore, muscle glycogen content at the onset of the exercise is crucial for maintaining not only endurance exercise but also short-term sprint exercise. On the sport fields, athletes are required to conduct practices or competitions for several times within a day. Therefore, it is important to recover muscle glycogen content after completion of exercise before the start of subsequent exercise. Betts et al. [6] revealed that recovery of muscle glycogen content following an exercise session was accelerated by an adequate quantity $(\sim 1 \mathrm{~g} /$ body mass $/ \mathrm{h})$ carbohydrate $\mathrm{CHO}$ intake. In contrast, insufficient $\mathrm{CHO}$ intake following an endurance exercise session delayed recovery of muscle glycogen content, while low CHO intake facilitated fat metabolism (e.g., increased plasma-free fatty acid concentration and decreased respiratory exchange ratio) compared with high $\mathrm{CHO}$ intake $[4,7,8]$. Thus, augmented fat metabolism during post-exercise period by insufficient $\mathrm{CHO}$ intake would reflect delayed recovery of muscle glycogen content. In addition, it may be associated with impaired endurance exercise performance during subsequent exercise. Therefore, assessment of fat metabolism during the early phase of post-exercise period is valuable.

Generally, fat metabolism can be evaluated using blood variables (e.g., plasma-free fatty acid, serum total ketone concentrations). However, due to an invasive fashion for evaluating these variables, development of non-invasive assessment for fat metabolism is required. Circulating free fatty acid is converted into acetyl-CoA via $\beta$-oxidation, and acetylCoA is transformed to ketone bodies in the liver. Ketone bodies consist of acetoacetate, 
3-hydroxybutanoic acid and acetone. Acetoacetate is initially produced, followed by 3hydroxybutanoic acid and acetone productions mediated by enzymatic degradation or decarboxylation, respectively [9]. Among ketone bodies, acetone translates to the lung and it is excreted as an exhaled gas due to its small molecular size [10]. Several studies have demonstrated that a positive correlation was observed between breath acetone level and serum beta-hydroxybutyrate concentration [11-14]. In addition, Anderson [10] suggested that increase in breath acetone level reflected augmented fat metabolism in the liver. Thus, assessment of breath acetone level can be an alternative and non-invasive procedure to evaluate changes in fat metabolism during post-exercise period $[15,16]$. Additionally, since acetone is produced from free fatty acid (FFA) which is facilitated by insufficient $\mathrm{CHO}$ intake [7], reduced $\mathrm{CHO}$ intake following an exercise session may rise breath acetone level during post-exercise period. However, no study has been conducted so far to clarify the effect of insufficient $\mathrm{CHO}$ intake after completion of an exhaustive exercise session on breath acetone level during post-exercise period. Therefore, the purpose of the present study was to determine the influence of low $\mathrm{CHO}$ intake following sprint exercise on breath acetone level. We hypothesized that reduced $\mathrm{CHO}$ intake following the sprint exercise would increase breath acetone level.

\section{Materials and Methods}

\subsection{Subjects}

Nine men (mean \pm standard error (SE): age, $20.8 \pm 0.2 \mathrm{yrs}$; height, $170.1 \pm 1.6 \mathrm{~cm}$; body mass, $65.1 \pm 1.8 \mathrm{~kg}$; BMI, $22.7 \pm 0.7 \mathrm{~kg} / \mathrm{m}^{2}$ ) were recruited. All subjects were healthy, and none of them have participated in any regular training program at the start of the experiment. Subjects were informed of the experimental procedures and possible risks involved in this study. Subsequently, an informed consent was obtained. The present study was approved was approved by the Ethics committee for Human Experiments at Ritsumeikan University, Japan.

\subsection{Experimental Overview}

All subjects visited the laboratory five times throughout the experiment. On the first visit, they conducted a familiarization session for repeated cycle sprint exercise. From the second to fifth visits, two main trials (consisting of two consecutive days, day 1 and day2) were performed. Each main trial consisted of either trial with consuming normal carbohydrate $(\mathrm{CHO})$ meal, following the repeated cycle sprint exercise (NOR trial), or with consuming reduced $\mathrm{CHO}$ meal, following the same exercise (LOW trial). These two trials were separated by at least 1 week, and the order of the trials was randomized. In addition, subjects were required to refrain from strenuous physical activity during $24 \mathrm{~h}$ prior to main trial on day1. The changes in breath acetone level, respiratory gas, and blood variables were compared until the following morning on day2 (Figure 1).

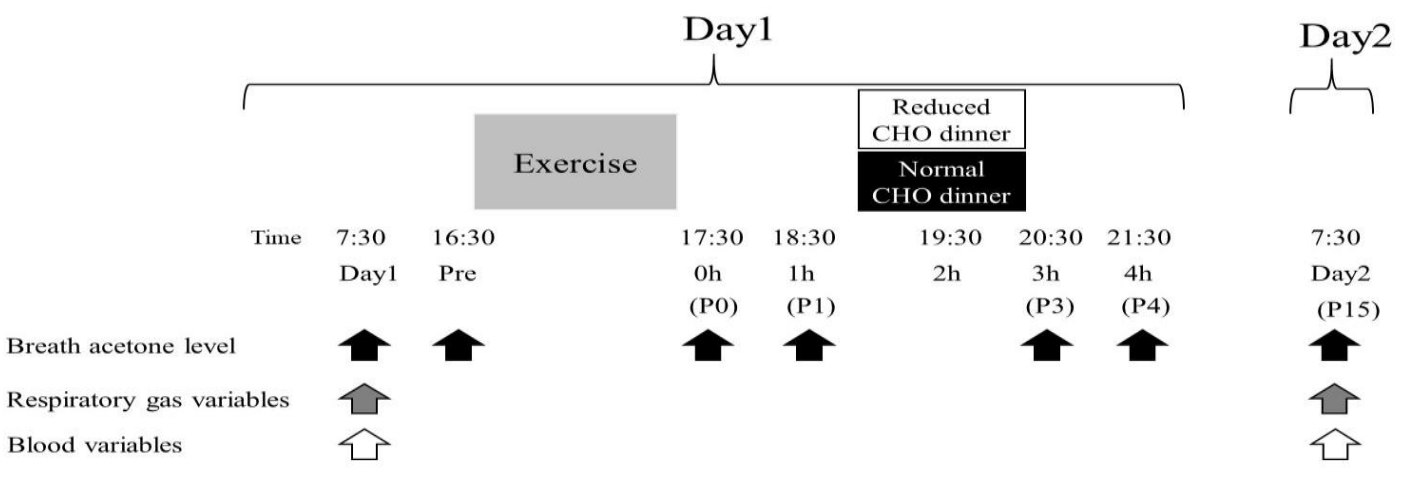

Figure 1. Experimental overview. 


\subsection{Familiarization Session}

About a week prior to commencing main trials (NOR trial or LOW trial), a familiarization session was performed. Subjects underwent submaximal cycling exercise at $50 \mathrm{~W}$ for $5 \mathrm{~min}$. They subsequently conducted $2 \times 3 \mathrm{~s}$ maximal cycle sprint exercise using an electromagnetically braked cycle ergometer (Power Max III; Konami Corp., Tokyo, Japan). The applied load was equivalent to $5 \%$ of body weight for the first cycle sprint exercise and $7.5 \%$ of body weight for the second cycle sprint exercise. After completing warm-up exercise, they performed $2 \times 30 \mathrm{~s}$ maximal cycle sprint exercise using an electromagnetically braked cycle ergometer. The applied load was set at $7.5 \%$ of body weight.

\subsection{Repeated Cycle Sprint Exercise Session on Day1 (Main Trial)}

On day1, the subjects came to the laboratory at 7:30, following an overnight fast (from 22:00 ). After completing the baseline measurements, the identical meal for breakfast was provided ( $461 \mathrm{kcal}: 77 \%$ for $\mathrm{CHO}, 5 \%$ for protein, $19 \%$ for fat). In addition, subjects consumed the prescribed lunch ( $821 \mathrm{kcal}: 64 \%$ for $\mathrm{CHO}, 15 \%$ for protein, $21 \%$ for fat). Any exercise aside from experiment-related exercise was not allowed throughout the day. At 16:30, they returned to the laboratory to start repeated cycle sprint exercise. As a warmup exercise, subjects initially performed submaximal cycling exercise for $5 \mathrm{~min}$. They subsequently conducted $2 \times 3 \mathrm{~s}$ maximal cycle sprint exercise using an electromagnetically braked cycle ergometer. The applied load was equivalent to $5 \%$ of body weight for the first cycle sprint exercise and $7.5 \%$ of body weight for the second cycle sprint exercise.

After completing warm-up exercise, subjects commenced main exercise. The main exercise consisted of $4 \times 30 \mathrm{~s}$ maximal cycle sprint exercise using an electromagnetically braked cycle ergometer. The applied load was equivalent to $7.5 \%$ of body weight. A $10 \mathrm{~min}$ of active rest was inserted between the sprints. This exercise protocol has been commonly used as maximal sprint exercise [17]. Subjects were verbally encouraged throughout each sprint to perform the exercise with maximal effort.

\subsection{Dietary Manipulation in NOR Trial and LOW Trial}

On day1, subjects consumed the isocaloric dinner with containing different $\mathrm{CHO}$ ratio (from 19:30, $2 \mathrm{~h}$ after completing exercise). In NOR trial, the meal was composed of 58\% for $\mathrm{CHO}, 14 \%$ for protein, $28 \%$ for fat. In contrast, the meal in LOW trial consisted of $18 \%$ for $\mathrm{CHO}, 27 \%$ for protein, $55 \%$ for fat. The energy intake was matched $1113 \mathrm{kcal}$ between meals. Dinner consisted of normal Japanese food (rice, potato salad, yogurt, Japanese omelet, Japanese fried chicken, tuna, and orange juice). After completing the dinner, the subjects stayed at accommodation located in the university. The sleep duration was unified from 23:00 to 07:00 for all subjects.

\subsection{Measurements}

In the morning on day1 and day2, breath acetone level, blood variables, and resting respiratory gas variables were evaluated, following an overnight fast. Breath acetone level was also evaluated immediately before and after exercise, $1 \mathrm{~h}, 3 \mathrm{~h}, 4 \mathrm{~h}$, and $15 \mathrm{~h}$ after completing repeated cycle sprint exercise. During the exercise, mean power output and maximal pedaling frequency were recorded. Moreover, heart rate was recorded throughout the exercise using a heart rate monitor (RCX5; POLAR corporation, Kempele, Finland).

\subsubsection{Breath Acetone Level}

Breath acetone level was evaluated using an automatic breath acetone analyzer (HOSIDEN corporation, Osaka, Japan). Subjects were required to exhale breath into automatic analyzer for $5 \mathrm{~s}$. In each measurement, breath acetone level was evaluated three times. The average value for three measurements was adopted. 


\subsubsection{Blood Variables}

In the morning on day1 and day2, venous blood samples were taken from an antecubital vain. Serum samples were subsequently obtained after a 10 -min centrifugation at $4{ }^{\circ} \mathrm{C}$ and stored at $-80{ }^{\circ} \mathrm{C}$ until analysis. From obtained serum samples, serum insulin, acetoacetic acid, 3-hydroxybutanoic acid, and total ketone concentrations were assayed at the clinical laboratory (SRL Inc., Tokyo, Japan). Further blood samples were obtained after each sprint for determinations of blood glucose and lactate concentrations. These concentrations were evaluated using a glucose analyzer (Free Style, Nipro Co., Osaka, Japan) and a lactate analyzer (Lactate Pro2; Arkray Co., Kyoto, Japan), respectively.

\subsubsection{Resting Respiratory Gas Variables}

In the morning on day 1 and day 2, subjects stayed rest for $10 \mathrm{~min}$ at the laboratory. Subsequently, respiratory gas samples were collected for 5 min using a breath-by-breath method and an automatic gas analyzer (AE300S; Minato Medical Science Co., Tokyo, Japan). The collected data were averaged every $30 \mathrm{~s}$ to determine respiratory exchange ratio (RER), oxygen consumption $\left(\mathrm{VO}_{2}\right)$, and carbon dioxide output $\left(\mathrm{VCO}_{2}\right)$. Substrate oxidation (fat and carbohydrate oxidation) was evaluated using the equation reported in a previous study [18].

\subsection{Statistical Analysis}

All values are presented as means \pm SE. Two-way repeated-measures analysis of variance (two-way ANOVA) was applied to assess the main effect (trial and time) and the interaction (trial $\times$ time). When the ANOVA revealed a significant interaction or main effect, the Tukey-Kramer test was performed to identify differences. In addition, paired $t$-test was used to compare resting respiratory gas and blood variables on day 1 and day 2 . A $p$-value $<0.05$ was considered significant for all measurements.

\section{Results}

\subsection{Power Output and Heart Rate During Repeated Sprint Exercise}

Maximal pedaling frequency, mean power output, and relative mean power output $(\mathrm{W} / \mathrm{kg})$ significantly decreased during set 3 and set 4 compared with set $1(p<0.05)$. However, no significant difference was observed for these variables between two trials (Table 1). Heart rate throughout the exercise session was not different significantly between two trials.

Table 1. Exercise performance variables during repeated sprint exercise.

\begin{tabular}{cccccc}
\hline & & Set 1 & Set 2 & Set 3 & Set 4 \\
\hline $\begin{array}{c}\text { Maximal pedaling frequency } \\
(\mathrm{rpm})\end{array}$ & NOR & $154 \pm 3$ & $153 \pm 2$ & $148 \pm 4^{*}$ & $144 \pm 3^{*}$ \\
\cline { 2 - 6 } & LOW & $154 \pm 3$ & $148 \pm 3$ & $145 \pm 2 *$ & $143 \pm 2 *$ \\
\hline $\begin{array}{c}\text { Mean power } \\
\text { output (W) }\end{array}$ & NOR & $612 \pm 20$ & $583 \pm 20$ & $556 \pm 21^{*}$ & $539 \pm 18^{*}$ \\
\cline { 2 - 6 } & LOW & $585 \pm 20$ & $575 \pm 18$ & $548 \pm 18^{*}$ & $537 \pm 19{ }^{*}$ \\
\hline $\begin{array}{c}\text { Relative mean power output } \\
(\mathrm{W} / \mathrm{KG})\end{array}$ & NOR & $9.4 \pm 0.4$ & $9.0 \pm 0.2$ & $8.5 \pm 0.2^{*}$ & $8.3 \pm 0.2^{*}$ \\
\cline { 2 - 6 } & LOW & $9.0 \pm 0.3$ & $8.8 \pm 0.2$ & $8.4 \pm 0.2^{*}$ & $8.2 \pm 0.2^{*}$ \\
\hline
\end{tabular}

Values are means \pm SE. ${ }^{*} p<0.05$ vs. Set1.

\subsection{Breath Acetone Level}

In NOR trial, breath acetone level was markedly decreased after consuming dinner until $4 \mathrm{~h}(\mathrm{P} 4)$ after exercise. Consequently, a significant reduction was observed at $3 \mathrm{~h}$ (P3) and $4 \mathrm{~h}$ (P4) compared with $1 \mathrm{~h}(\mathrm{P} 1)$ in NOR trial $(p<0.05)$. At $4 \mathrm{~h}$ after exercise, LOW trial showed significantly higher breath acetone level than that in NOR trial $(p<0.05)$. However, 
there was no significant difference in breath acetone level between trials in the morning on day2 (Figure 2).

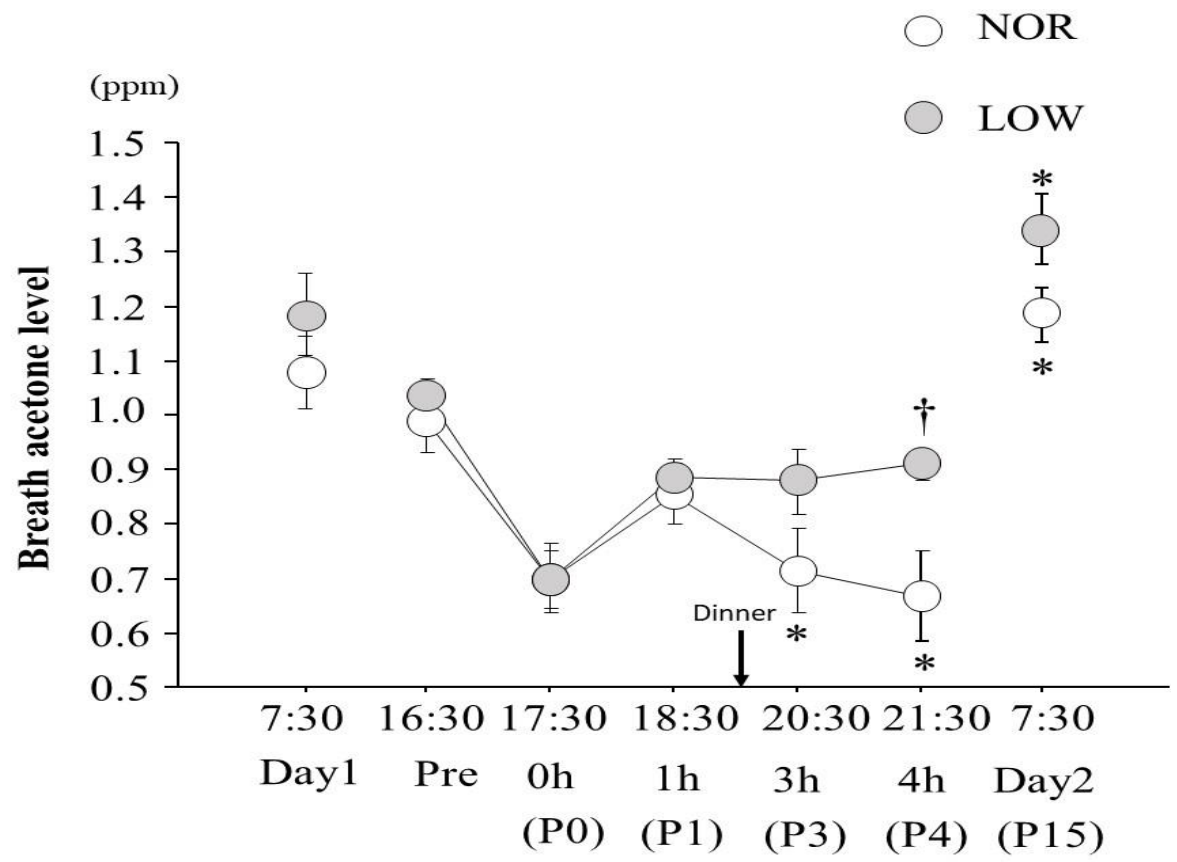

Figure 2. Changes in breath acetone level. Values are means \pm SE. ${ }^{*} p<0.05$ vs. $1 \mathrm{~h},+p<0.05$ vs. trial.

\subsection{Blood Variables}

Blood lactate concentration was increased significantly with exercise, but no significant difference was found between the trials. In addition, blood glucose concentration was not different significantly between the two trials (Table 2).

Table 2. Blood lactate and glucose concentrations.

\begin{tabular}{ccccccccc}
\hline & & Day1 & Pre & Set1 & Set2 & Set3 & Set4 & Day2 \\
\hline $\begin{array}{c}\text { Lactate } \\
(\mathrm{mmol} / \mathrm{L})\end{array}$ & NOR & $1.3 \pm 0.1$ & $1.2 \pm 0.1$ & $8.1 \pm 0.6^{*}$ & $13.2 \pm 0.8^{*}$ & $17.4 \pm 0.8^{*}$ & $19.8 \pm 1^{*}$ & $1.2 \pm 0.1$ \\
\cline { 2 - 9 } & LOW & $1.4 \pm 0.1$ & $1.4 \pm 0.1$ & $10.8 \pm 0.8^{*}$ & $13.8 \pm 0.5^{*}$ & $15.6 \pm 0.7^{*}$ & $17.6 \pm 0.9^{*}$ & $1.1 \pm 0.1$ \\
\hline $\begin{array}{c}\text { Glucose } \\
(\mathrm{mg} / \mathrm{dL})\end{array}$ & NOR & $83 \pm 2$ & $90 \pm 3$ & $85 \pm 3$ & $86 \pm 3$ & $92 \pm 4$ & $92 \pm 4$ & $81 \pm 1$ \\
\cline { 2 - 9 } & LOW & $85 \pm 2$ & $88 \pm 2$ & $86 \pm 3$ & $87 \pm 3$ & $89 \pm 4$ & $91 \pm 3$ & $81 \pm 1$ \\
\hline
\end{tabular}

Values are means \pm SE. ${ }^{*} p<0.05$ vs. Pre.

Figure 3 presents changes in serum 3-hydroxybutanoic acid (A) and total ketone body concentrations (B) in the morning on day1 and day2. On day1, no significant difference was observed between two trials for serum 3-hydroxybutanoic acid or total ketone body concentrations. In contrast, both variables were significantly higher in LOW trial than in NOR trial on day2 $(p<0.05)$. Furthermore, serum acetoacetic acid concentration was significantly higher in LOW trial on day2 $(p<0.05)$. Serum insulin concentration was not different significantly between the trials on day1 and day 2 . 

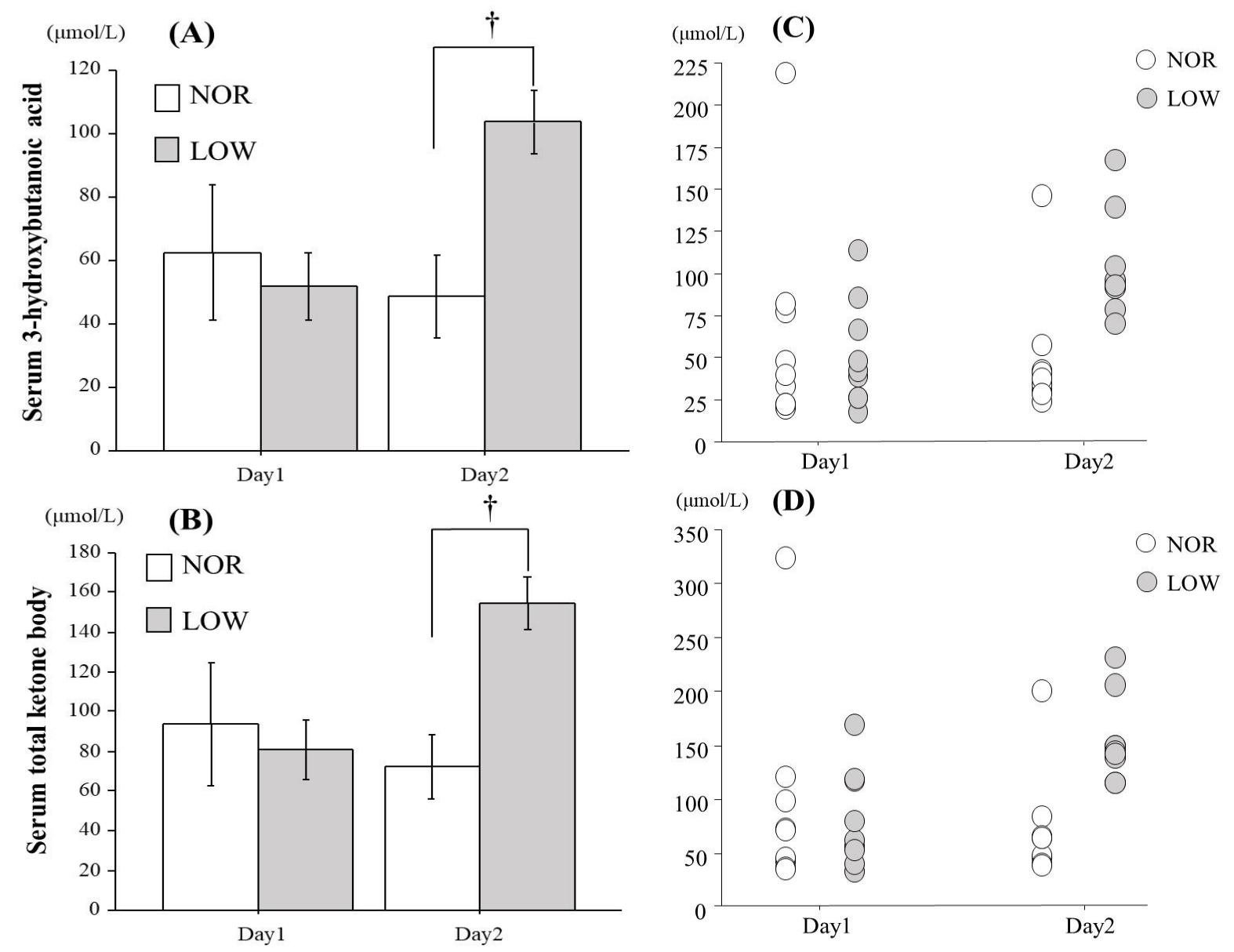

Figure 3. Changes in mean value of serum 3-hydroxybutanoic acid (A), total ketone body concentration (B), individual value of serum 3-hydroxybutanoi cacid (C) and total ketone body concentration (D). Values are means \pm SE. $\dagger p<0.05$ vs. trial.

\subsection{Resting Respiratory Gas Variables}

$\dot{\mathrm{V}} \mathrm{O}_{2}$ and $\dot{\mathrm{V}} \mathrm{CO}_{2}$ did not differ significantly between two trials in the morning on day1 and day2. There was no significant difference in RER between two trials in the morning on day1. However, LOW trial showed significantly lower RER in the morning on day2 $(p<0.05)$. In addition, $\mathrm{CHO}$ oxidation was significantly lower in the LOW trial than in NOR trial on day2, whereas LOW trial presented significantly higher fat oxidation $(p<0.05$, Figure 4). 

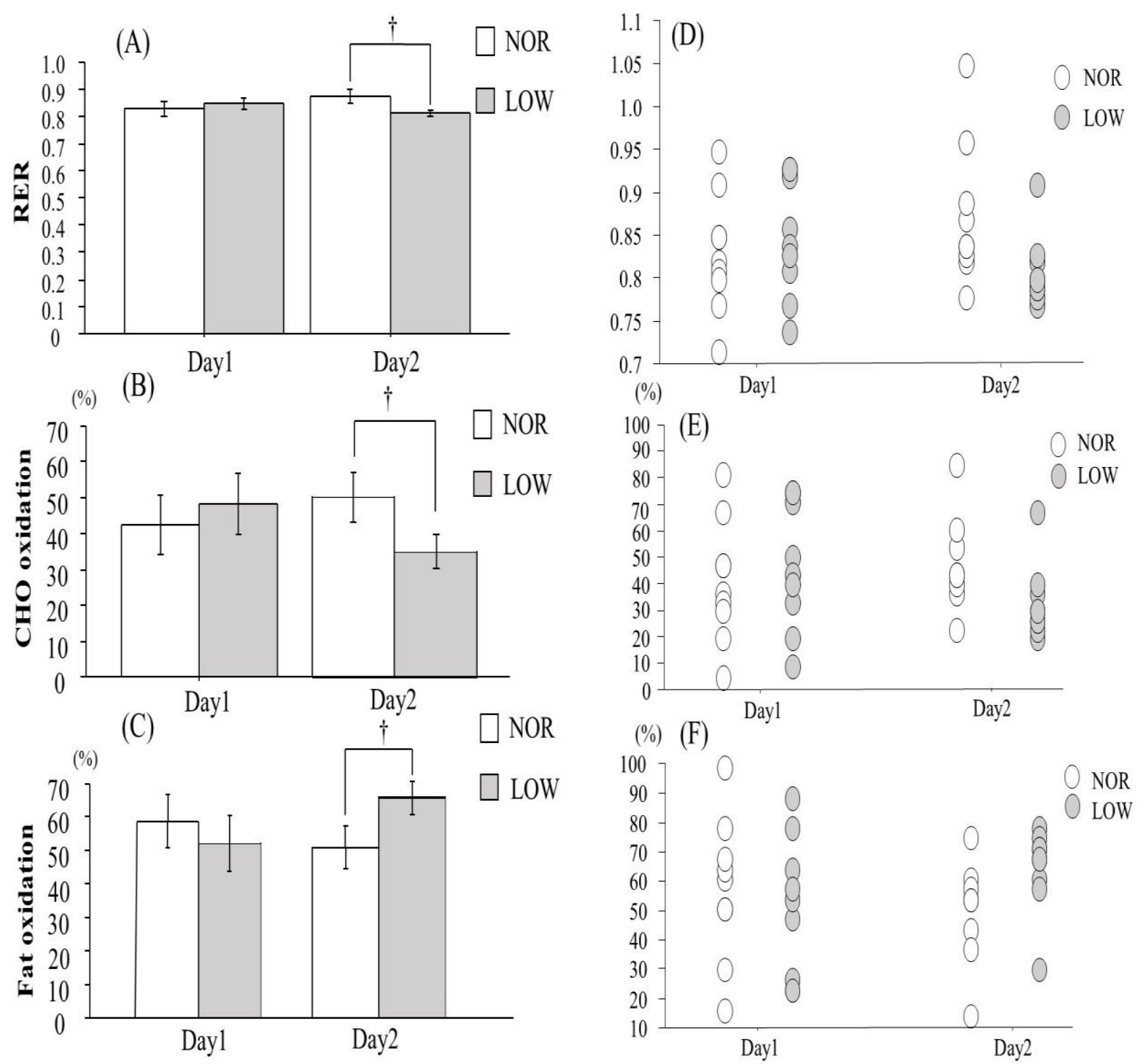

Figure 4. Changes in mean value of RER (A), CHO oxidation (B), fat oxidation (C), individual value ofRER (D), CHO oxidation (E) and fat oxidation (F). Values are means \pm SE. $+p<0.05$ vs. trial.

\section{Discussion}

The present study investigated the effect of $\mathrm{CHO}$ intake following an acute sprint exercise session on breath acetone level. The main finding was that breath acetone level following normal $\mathrm{CHO}$ intake after the sprint exercise (NOR trial) markedly decreased until $4 \mathrm{~h}$ after the completion of exercise. Consequently, LOW trial showed significantly higher breath acetone level compared with NOR trial $4 \mathrm{~h}$ after completing the exercise. These findings indicate that breath acetone was strongly affected by $\mathrm{CHO}$ intake during early phase of post-exercise.

Mean power output and maximal pedaling frequency during repeated sprint exercise did not differ significantly between the two trials. Additionally, post-exercise blood lactate concentration was not significantly different between the conditions. Therefore, it appeared that sprint exercise in both trials evoked similar physiological stimulus. Catecholamine and growth hormone $(\mathrm{GH})$ promote lipolysis. In addition, exercise-induced elevation of these hormones are highly dependent on exercise intensity [19]. In the present study, since maximal cycle sprint exercise was applied, it appeared that exercise-induced catecholamine and GH elevations were maximized. Moreover, both trials presented comparable increase 
in breath acetone level $1 \mathrm{~h}$ after completing exercise ( $1 \mathrm{~h}$ before consuming the dinner). Therefore, exercise-induced increase in lipolysis would be similar between two trials. In the morning on day2, serum 3-hydroxybutanoic acid and total ketone body concentrations were significantly higher in LOW trial than in NOR trial. Furthermore, fat oxidation at rest on day2 was significantly higher in LOW trial compared with NOR trial, indicating that fat metabolism in the morning on day 2 was facilitated in LOW trial. Delayed recovery of muscle glycogen content during post-exercise period might be a reason for augmented fat metabolism in LOW trial. Previous studies showed that recovery of glycogen content following an exhaustive exercise was faster in liver than in muscle [20-22]. In addition, reduced $\mathrm{CHO}$ intake during post-exercise delayed recovery of muscle glycogen content $[4,23]$. In addition, insufficient $\mathrm{CHO}$ intake led to increase in FFA concentration [4,7]. Although we did not assess glycogen content in either muscle or liver, it is plausible that recovery of muscle glycogen content was delayed due to low $\mathrm{CHO}$ intake following sprint exercise on day1. Therefore, reduced $\mathrm{CHO}$ intake following sprint exercise augmented fat metabolism the next morning.

Somewhat surprisingly, breath acetone level did not differ significantly between two trials in the morning on day2. Fasted status has shown to increase breath acetone level $[14,24]$. In the present study, subjects were not allowed to take meals after consuming dinner following the exercise on day1. Therefore, potential difference in breath acetone level between the two trials may be masked due to an overnight fasting. However, it was notable to find that breath acetone level $4 \mathrm{~h}$ after completion of exercise ( $2 \mathrm{~h}$ after consuming meal) was significantly higher in LOW trial than in NOR trial. Bovey et al. [25] compared breath acetone level following high $\mathrm{CHO}$ (HC trial) and low $\mathrm{CHO}$ (LC trial) intake. Consequently, $\mathrm{HC}$ trial presented a significant reduction of breath acetone level $1 \mathrm{~h}$ after consuming meal. On the other hand, LC trial showed significantly higher breath acetone level compared with $\mathrm{HC}$ trial $2 \mathrm{~h}$ after consuming the meal. The authors suggested that increase in breath acetone level was abolished by $\mathrm{CHO}$ intake.

The present study includes several limitations. Firstly, exercise performance was not evaluated in the morning on day2. Therefore, we are not able to present potential relations between breath acetone level during post-exercise period and recovery of exercise performance. There is a possibility that muscle glycogen content was not recovered to baseline level in LOW trial, which may impair endurance exercise performance. Additionally, since exercise with low muscle glycogen content causes compensatory increase in fat metabolism [26], higher breath acetone level (i.e., augmented fat metabolism) may reflect attenuated exercise performance. Thirdly, we did not evaluate change in serum total ketone concentration after consuming the meal on day1. Thus, relationship between breath acetone level and serum total ketone concentration after consuming the meals has not been clarified. Fourthly, we did not include a fasting trial (without consuming the meal) following exercise. The control trial without consuming the meal will be important in future work to determine breath acetone level in the fasting state following an exhaustive exercise. Finally, the present study utilized repeated cycle sprint exercise. Therefore, further determination of changes in breath acetone level following different types of exercise protocols (e.g., prolonged endurance exercise, team-based sports with 80-90 min of match play) would be informative.

\section{Conclusions}

A significant higher breath acetone level was observed in LOW trial than in NOR trial $4 \mathrm{~h}$ after completing exercise. However, breath acetone level did not differ significantly between two trials in the morning on day2. These findings indicate that breath acetone level during early phase of post-exhaustive exercise is affected by $\mathrm{CHO}$ intake.

Author Contributions: The present study was designed by N.O. and K.G.; data was collected and analyzed by N.O., H.I., and K.G., data interpretation and manuscript presentation were undertaken by N.O., H.I., and K.G. All authors approved the final version of the paper. All authors have read and agreed to the published version of the manuscript. 
Funding: This research was funded by [Japan Society for the Promotion of Science] grant number [18K19763].

Institutional Review Board Statement: The study was conducted according to the guidelines of the Declaration of Helsinki, and approved by the Ethics Committee for Human Experiments of Ritsumei kan University (protocol code BKC-2018-028, approved in 8 November 2019), Japan.

Informed Consent Statement: Informed consent was obtained from all subjects involved in the study. Data Availability Statement: Data is contained with in the article.

Acknowledgments: We thank all subjects for cooperation with this research. We also appreciate to the laboratory members for technical support during the experiment.

Conflicts of Interest: The authors declare no conflict of interest.

\section{References}

1. Holloszy, J.O.; Kohrt, W.M. Regulation of carbohydrate and fat metabolism during and after exercise. Annu. Rev. Nutr. 1996, 16, 121-138. [CrossRef] [PubMed]

2. Bergström, J.; Hermansen, L.; Hultman, E.; Saltin, B. Diet, muscle glycogen and physical performance. Acta Physiol. Scand. 1967, 71, 140-150. [CrossRef] [PubMed]

3. Temesi, J.; Johnson, N.A.; Raymond, J.; Burdon, C.A.; O'Connor, H.T. Carbohydrate ingestion during endurance exercise improves performance in adults. J. Nutr. 2011, 141, 890-897. [CrossRef] [PubMed]

4. Alghannam, A.F.; Jedrzejewski, D.; Tweddle, M.G.; Gribble, H.; Bilzon, J.; Thompson, D.; Tsintzas, K.; Betts, J.A. Impact of Muscle Glycogen Availability on the Capacity for Repeated Exercise in Man. Med. Sci. Sports Exerc. 2016, 48, 123-131. [CrossRef]

5. Bogdanis, G.C.; Nevill, M.E.; Boobis, L.H.; Lakomy, H.K.; Nevill, A.M. Recovery of power output and muscle metabolites following $30 \mathrm{~s}$ of maximal sprint cycling in man. J. Physiol. 1995, 482, 467-480. [CrossRef]

6. Betts, J.A.; Williams, C. Short-term recovery from prolonged exercise: Exploring the potential for protein ingestion to accentuate the benefits of carbohydrate supplements. Sports Med. 2010, 40, 941-959. [CrossRef]

7. Tsintzas, K.; Williams, C.; Boobis, L.; Symington, S.; Moorehouse, J.; Garcia-Roves, P.; Nicholas, C. Effect of carbohydrate feeding during recovery from prolonged running on muscle glycogen metabolism during subsequent exercise. Int. J. Sports Med. 2003, 24, 452-458. [CrossRef]

8. Wong, S.H.; Williams, C. Influence of different amounts of carbohydrate on endurance running capacity following short term recovery. Int. J. Sports Med. 2000, 21, 444-452. [CrossRef]

9. Qiao, Y.; Gao, Z.; Liu, Y.; Cheng, Y.; Yu, M.; Zhao, L.; Duan, Y.; Liu, Y. Breath ketone testing: A new biomarker for diagnosis and therapeutic monitoring of diabetic ketosis. BioMed Res. Int. 2014, 2014, 869186. [CrossRef]

10. Anderson, J.C. Measuring breath acetone for monitoring fat loss: Review. Obesity 2015, 23, 2327-2334. [CrossRef]

11. Hancock, G.; Sharma, S.; Galpin, M.; Lunn, D.; Megson, C.; Peverall, R.; Richmond, G.; Ritchie, G.A.D.; Owen, K.R. The correlation between breath acetone and blood betahydroxybutyrate in individuals with type 1 diabetes. J. Breath Res. 2020, 15. [CrossRef] [PubMed]

12. Tanda, N.; Hinokio, Y.; Washio, J.; Takahashi, N.; Koseki, T. Analysis of ketone bodies in exhaled breath and blood of ten healthy Japanese at OGTT using a portable gas chromatograph. J. Breath Res. 2014, 8, 046008. [CrossRef] [PubMed]

13. Samudrala, D.; Lammers, G.; Mandon, J.; Blanchet, L.; Schreuder, T.H.A.; Hopman, M.T.; Harren, F.J.H.; Tappy, L.; Cristescu, S.M. Breath acetone to monitor life style interventions in field conditions: An exploratory study. Obesity 2014, 22, 980-983. [CrossRef] [PubMed]

14. Saasa, V.; Beukes, M.; Lemmer, Y.; Mwakikunga, B. Blood Ketone Bodies and Breath Acetone Analysis and Their Correlations in Type 2 Diabetes Mellitus. Diagnostics 2019, 9, 244. [CrossRef] [PubMed]

15. Güntner, A.T.; Kompalla, J.F.; Landis, H.; Theodore, S.J.; Geidl, B.; Sievi, N.A.; Kohler, M.; Pratsinis, S.E.; Gerber, P.A. Guiding Ketogenic Diet with Breath Acetone Sensors. Sensors 2018, 18, 3655. [CrossRef]

16. Hori, A.; Ichihara, M.; Kimura, H.; Ogata, H.; Kondo, T.; Hotta, N. Inhalation of molecular hydrogen increases breath acetone excretion during submaximal exercise: A randomized, single-blinded, placebo-controlled study. Med. Gas. Res. 2020, 10, 96-102. [CrossRef]

17. Kasai, N.; Kojima, C.; Sumi, D.; Takahashi, H.; Goto, K.; Suzuki, Y. Impact of 5 Days of Sprint Training in Hypoxia on Performance and Muscle Energy Substances. Int. J. Sports Med. 2017, 38, 983-991. [CrossRef]

18. Manetta, J.; Brun, J.F.; Perez-Martin, A.; Callis, A.; Prefaut, C.; Mercier, J. Fuel oxidation during exercise in middle-aged men: Role of training and glucose disposal. Med. Sci. Sports Exerc. 2002, 34, 423-429. [CrossRef]

19. Lundsgaard, A.M.; Fritzen, A.M.; Kiens, B. The Importance of Fatty Acids as Nutrients during Post-Exercise Recovery. Nutrients 2020, 12, 280. [CrossRef]

20. Casey, A.; Mann, R.; Banister, K.; Fox, J.; Morris, P.G.; Macdonald, I.A.; Greenhaff, P.l. Effect of carbohydrate ingestion on glycogen resynthesis in human liver and skeletal muscle, measured by (13)C MRS. Am. J. Physiol. Endocrinol. Metab. 2000, 278, E65-E75. [CrossRef] 
21. Gonzalez, J.T.; Fuchs, C.J.; Betts, J.A.; van Loon, L.J. Liver glycogen metabolism during and after prolonged endurance-type exercise. Am. J. Physiol. Endocrinol. Metab. 2016, 311, E543-E553. [CrossRef] [PubMed]

22. Fuchs, C.J.; Gonzalez, J.T.; Beelen, M.; Cermak, N.M.; Smith, F.E.; Thelwall, P.E.; Taylor, R.; Trenell, M.I.; Stevenson, E.J.; van Loon, L.J.C. Sucrose ingestion after exhaustive exercise accelerates liver, but not muscle glycogen repletion compared with glucose ingestion in trained athletes. J. Appl. Physiol. 2016, 120, 1328-1334. [CrossRef] [PubMed]

23. Burke, L.M.; van Loon, L.J.C.; Hawley, J.A. Postexercise muscle glycogen resynthesis in humans. J. Appl. Physiol. 2017, 122, 1055-1067. [CrossRef] [PubMed]

24. Güntner, A.T.; Sievi, N.A.; Theodore, S.J.; Gulich, T.; Kohler, M.; Pratsinis, S.E. Noninvasive Body Fat Burn Monitoring from Exhaled Acetone with Si-doped $\mathrm{WO}_{3}$-sensing Nanoparticles. Anal. Chem. 2017, 89, 10578-10584. [CrossRef]

25. Bovey, F.; Cros, J.; Tuzson, B.; Seyssel, K.; Schneiter, P.; Emmenegger, L.; Tappy, L. Breath acetone as a marker of energy balance: An exploratory study in healthy humans. Nutr. Diabetes 2018, 8, 50. [CrossRef]

26. Margolis, L.M.; Wilson, M.A.; Whitney, C.C.; Carrigan, C.T.; Murphy, N.E.; Hatch, A.M.; Montain, S.J.; Pasiakos, S.M. Exercising with low muscle glycogen content increases fat oxidation and decreases endogenous, but not exogenous carbohydrate oxidation. Metabolism 2019, 97, 1-8. [CrossRef] 